\title{
Differentiation of rat adipose-derived mesenchymal stem cells into corneal-like epithelial cells driven by PAX6
}

\author{
JING SUN ${ }^{1,2^{*}}$, WEI-HUA LIU ${ }^{3}$, FENG-MEI DENG $^{2 *}$, YONG-HUI LUO $^{3}$, KE WEN $^{3}$, \\ HONG ZHANG ${ }^{3}$, HAI-RONG LIU ${ }^{3}$, JIANG WU ${ }^{4}$, BING-YIN SU ${ }^{2}$ and YI-LUN LIU ${ }^{3}$
}

\begin{abstract}
${ }^{1}$ Department of Neurobiology, Chongqing Key Laboratory of Neurobiology, Third Military Medical University, Chongqing 400038; ${ }^{2}$ Department of Regeneration Key Lab of Sichuan Province, Chengdu Medical College; ${ }^{3}$ Department of Surgery, The First Affiliated Hospital of Chengdu Medical College, Chengdu, Sichuan 610500; ${ }^{4}$ Department of Biomedical

Engineering, West China Center of Medical Sciences, Sichuan University, Chengdu, Sichuan 610041, P.R. China
\end{abstract}

Received March 7, 2017; Accepted September 6, 2017

DOI: $10.3892 / e t m .2017 .5576$

\begin{abstract}
Corneal integrity, transparency and vision acuity are maintained by corneal epithelial cells (CECs), which are continuously renewed by corneal limbal stem cells (LSCs). Deficiency of CECs and/or LSCs is associated with numerous ocular diseases. Paired box (PAX)6 is an eye development-associated transcription factor that is necessary for cell fate determination and differentiation of LSCs and CECs. In the present study, the PAX6 gene was introduced into adipose-derived rat mesenchymal stem cells (ADMSCs) to investigate whether PAX6-transfected cells were able to transdifferentiate into corneal-like epithelial cells and to further verify whether the cells were suitable as a cell source for corneal transplantation. The ADMSCs were isolated from the bilateral inguinal region of healthy Sprague Dawley rats. The characteristics of ADMSCs were identified using flow cytometric analysis. After subculture, ADMSCs underwent transfection with recombinant plasmid containing either PAX6-enhanced green fluorescent protein (EGFP) complementary (c)DNA or EGFP cDNA (blank plasmid group), followed by selection with G418 and determination of the transfection efficiency. Subsequently, the morphology of the ADMSCs and the expression profiles of corneal-specific markers CK3/12 and epithelial-specific adhesion protein were
\end{abstract}

Correspondence to: Dr Bing-Yin Su, Department of Regeneration Key Lab of Sichuan Province, Chengdu Medical College, 783 Xindu Avenue, Chengdu, Sichuan 610500, P.R. China

E-mail: subingyinn@163.com

Dr Yi-Lun Liu, Department of Surgery, The First Affiliated Hospital of Chengdu Medical College, 278 Bao Guang Avenue, Chengdu, Sichuan 610500, P.R. China

E-mail: yilunliu@foxmail.com

*Contributed equally

Key words: adipose-derived mesenchymal stem cells, corneal-like epithelial cells, paired box 6 determined. E-cadherin was detected using immunofluorescence staining and western blot analysis at 21 days following transfection. An MTT cell proliferation and a colony formation assay were performed to assess the proliferative activity and clonogenicity of PAX6-transfected ADMSCs. Finally, the PAX6-expressing ADMSCs were transplanted onto the cornea of a rabbits with limbal stem cell deficiency (LSCD). At 21 days after transfection, the ADMSCs with PAX6 transfection exhibited a characteristic flagstone-like appearance with assembled corneal-like epithelial cells, and concomitant prominent expression of the corneal-specific markers cytokeratin 3/12 and E-cadherin. Furthermore, the proliferation and colony formation ability of PAX6-overexpressing ADMSCs was significantly retarded. The transplantation experiment indicated that PAX6-reprogramed ADMSCs attached to and replenished the damaged cornea via formation of stratified corneal epithelium. Taken together, these results suggested that conversion of ADMSCs into corneal-like epithelium may be driven by PAX6 transfection, which makes ADMSCs a promising cell candidate for the treatment of LSCD.

\section{Introduction}

The surface of the cornea consists of a unique type of non-keratinized epithelial cells [corneal epithelial cells (CECs)] that is essential to assure corneal transparency and acuity of vision. CECs are continuously renewed by a population of stem cells or progenitor cells that reside in the corneal limbus. However, severe corneal injury caused by chemical or mechanical injury, and immune or hereditary diseases may lead to corneal inflammation, ulceration, neovascularization and limbal stem cell deficiency (LSCD), all of which may cause blindness (1-3). Although corneal transplantation remains the most effective method for corneal damage, graft rejection remains the major cause of its failure. The application of allogenic or autologous limbal transplantation is also limited due to significant risk of systemic immunosuppression or compromising the contralateral normal eye (4-6). Therefore, looking for a safe and sufficient cell source for the bioengineered corneal epithelium is an urgent issue at present. 
A new modality to reconstruct the ocular surface by using bioengineered CECs has emerged within the last decade, and the application of mesenchymal stem cells (MSCs) as bioengineered corneal epithelia has been attempted in numerous experimental studies $(7,8)$. Studies have confirmed that corneal epithelial-like cells may be generated from bone marrow mesenchymal and embryonic stem cells (ESCs) $(9,10)$. Numerous potential advantages of adipose-derived MSCs (ADMSCs) including easy accessibility, low immunogenicity, potent pluripotency, the lack of ethical controversies and a reduced risk of contamination have made them a particularly attractive source of seed cells for bioengineered corneal epithelium (11-14).

Paired box 6 (PAX6) is a member of the PAX gene transcription factor family that is an earlier differentiation marker expressed by CECs, and the main driver of the differentiation of CECs (15-17). PAX6 is required not only for embryonic but also for postnatal development, as well as the maintenance of the adult cornea $(18,19)$. It has been demonstrated that PAX6-transfected ESCs differentiate into corneal epithelial-like cells in vitro (20). In this regard, the present study speculated that preferential differentiation of ADMSCs into corneal epithelial-like cells may also be induced by PAX6 transfection. Preliminary results were obtained by a previous study on mice by our group, which proved morphologically that ADMSCs derived from mice transform into corneal epithelial-like cells following PAX6 transfection.

In the present study, the differentiation capacity of ADMSCs into corneal epithelial-like cells driven by PAX6 was investigated in vitro. Furthermore, the therapeutic and repair potential of ADMSCs with engineered expression of PAX6 for corneal epithelial defects was tested in vivo in a rabbit model of LSCD. The results of the present study confirmed that engineered ADMSCs may serve as an ideal type of seed cell for the treatment of LSCD disease.

\section{Materials and methods}

Main reagents. Dulbecco's modified Eagle's medium (DMEM)/F12, 10\% fetal bovine serum (FBS) and type I collagenase were purchased from Sigma-Aldrich (Merck KGaA, Darmstadt, Germany) Anti-rat CD34-Fluorescein is othiocyanate (FITC; cat no. sc-7324) was purchased from Santa Cruz Biotechnology, Inc. (Santa Cruz, CA, USA); CD90-FITC (cat no. 206105) and CD45-FITC (cat no. 202205) were purchased from BioLegend Inc. (San Diego, CA, USA); and CD105-Phycoerythrin (PE; cat no. FAB10971P) was purchased from BD Biosciences (Franklin Lakes, NJ, USA).

Rabbit anti-PAX6 polyclonal antibody (cat no. ab5790), mouse anti-cytokeratin (CK) 3/12 monoclonal antibody (cat no. ab68260) and mouse anti-E-cadherin (cat no. ab76055) were obtained from Abcam (Cambridge, MA, USA). Horseradish peroxidase-conjugated mouse anti-rabbit (cat no. sc-2357) and donkey anti-mouse antibody (cat no. sc-2314) were purchased from Santa Cruz Biotechnology. PAX6-enhanced green fluorescence protein (EGFP) complementary (c)DNA plasmid [item no. CCS-Mm04345-PCDNA3.1(+)] was constructed by Guangzhou FulenGen Co., Ltd. (Guangzhou, China), where the PAX6 gene inserted had a GenBank accession no. of
NM_013627 and an open reading frame length of 1,311 bp (Fig. 1). The Endo-free Plasmid Mini Kit II was purchased from Omega Bio-tek (cat no. D6950-02B; Norcross, GA, USA). Lipofectamine ${ }^{\circledR} 2000$ transfection reagent (cat no. 11668-019) and Opti-MEM ${ }^{\circledR}$ I Reduced Serum Medium (cat no. 31985-070) were from Invitrogen (Carlsbad, CA, USA). The RNeasy Plus Mini Kit was obtained from Qiagen (cat no. 74134; Hilden, Germany). The PrimeScript ${ }^{\circledR}$ RT reagent kit (cat no. RR047A) and the SYBR Premix Ex Taq ${ }^{\mathrm{TM}}$ kit (cat no. RR420A) was purchased from Takara Biotechnology (Otsu, Japan).

Ethics statement. This study was approved by the Animal Experimentation Ethics Committee of Chengdu Medical College (Chengdu, China). Animal experiments were performed in a manner consistent with the Statement for the Use of Animals in Ophthalmic and Vision Research.

Animals. A total of 20 male Sprague Dawley (SD) rats aged 6 weeks with a mean body weight of $220 \pm 10 \mathrm{~g}$ and 10 male New Zealand white rabbits aged 6 months with an initial body weight of $2.0 \pm 0.4 \mathrm{~kg}$ were purchased from the Experimental Animal Center of Sichuan University (Chengdu, China). The animals were housed at an ambient temperature of $25 \pm 2^{\circ} \mathrm{C}$ and a relative humidity of $65-70 \%$ under a 12-h light/dark cycle. All animals were fed according to the Specific Pathogen Free Animal Criteria, and were allowed free access to food and water.

Isolation, cultivation and identification of ADMSCs. The ADMSCs were isolated from bilateral inguinal fat pads of healthy SD rats. Adipose tissue were washed extensively with sterile PBS and minced into pieces of $2 \mathrm{~mm}^{3}$ with fine scissors. The tissue was digested with $0.1 \%$ type I collagenase for $1 \mathrm{~h}$ at $37^{\circ} \mathrm{C}$. After partial digestion, the collagenase activity was neutralized by adding DMEM/F12 supplemented with $10 \%$ FBS followed by centrifugation at $500 \mathrm{x}$ g for $10 \mathrm{~min}$ at $4^{\circ} \mathrm{C}$. The resulting cell suspension was filtered through a $100-\mathrm{mm}$ cell strainer and centrifuged at $500 \mathrm{x}$ g for $5 \mathrm{~min}$ at $4^{\circ} \mathrm{C}$. Cell pellets were resuspended in DMEM/F12 supplemented with $10 \% \mathrm{FBS}$, and incubated at $37^{\circ} \mathrm{C}$ in a $5 \% \mathrm{CO}_{2}$ incubator. After 72-96 h, non-adherent cells were discarded and the medium was changed every 2-3 days. Cells were maintained in culture until they reached $80 \%$ confluence and were examined daily using an inverted fluorescence microscope. The ADMSCs at the third passage were trypsinized, washed with PBS and incubated with antibodies against CD34, CD45, CD90 and CD105(1:100) for $30 \mathrm{~min}$ at $4^{\circ} \mathrm{C}$. Flow cytometry detection was performed using a BD Accuri C6 flow cytometer (BD Biosciences).

Stable transfection with PAX6. ADMSCs at the third passage were seeded into 24 -well plates at a density of $0.8 \times 10^{5}$ cells/well, setting 3 replicates for each cell clone. After being cultured for $24 \mathrm{~h}$ in antibiotic-free DMEM/F12 containing 10\% FBS, the cells were $\sim 95 \%$ confluent. The cultured ADMSCs were respectively subjected to transfection with recombinant PAX6-EGFP cDNA or EGFP cDNA plasmid using Lipofectamine ${ }^{\circledR} 2000$ transfection reagent according to the manufacturer's instructions. The cells were transfected at $37^{\circ} \mathrm{C}$ in a $\mathrm{CO}_{2}$ incubator for $24 \mathrm{~h}$ prior to testing for transgene expression. The cells 

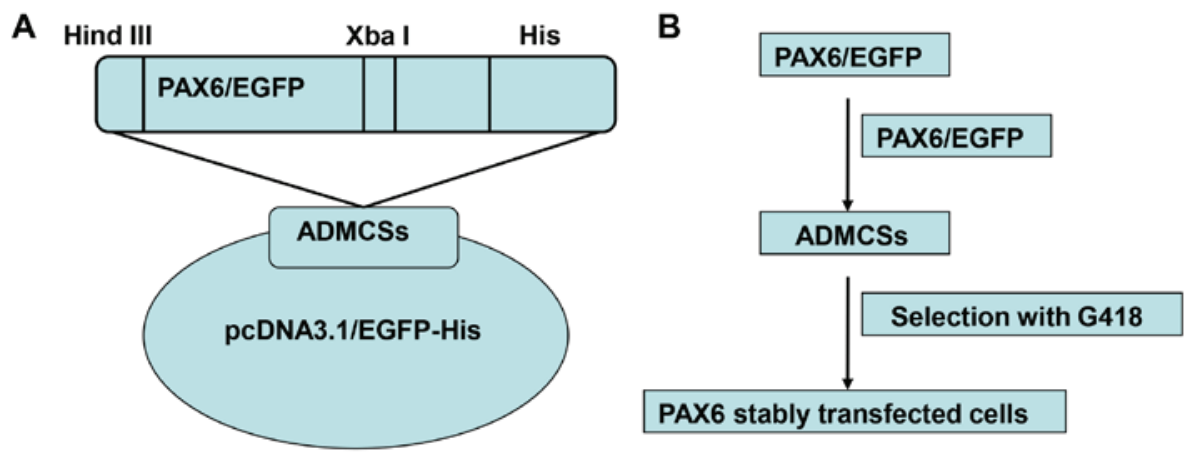

Figure 1.(A) Map of the construction protocol for the PAX6/EGFP-His expression plasmid.PAX6-EGFP cDNA plasmid [item no.CCS-Mm04345-PCDNA3.1(+)] was constructed by Guangzhou FulenGen Co., Ltd. The PAX6-EGFP fusion gene void of the termination codon of the PAX6 gene was inserted between the HindIII and XbaI sites in the backbone of the pCDNA3.1/His vector. The pCDNA3.1/His vector contained a neomycin-resistance gene for G418 selection with SV40 as the driver. The PAX6 gene had a primary GenBank accession no. of NM_013627 and an open reading frame length of 1,311 bp. (B) Schematic of the experimental protocol. ADMSCs were transfected with the PAX6 gene using Lipofectamine. The transfected cells were cultivated with G418-containing media for 7 days. The resultant stably PAX6 expression vector-transfected cells were transplanted onto the cornea of a rabbit limbal stem cell deficiency model. Seven days after transplantation, the repair potential of the PAX6-reprogramed ADMSCs for corneal epithelial defects was evaluated. EGFP, enhanced green fluorescence protein; PAX6, paired box 6; ADMSCs, adipose-derived mesenchymal stem cells.

were then passaged at 1:10 dilution in fresh growth medium. The following day, the cells were selected in G418-containing medium $(600 \mu \mathrm{g} / \mathrm{ml})$ for 7 days. Thirty days later, when cell death ceased and proliferation occurred thereafter, $800 \mu \mathrm{g} / \mathrm{ml}$ G418 was replaced with $400 \mu \mathrm{g} / \mathrm{ml} \mathrm{G} 418$ for maintenance. After 14 days, the stable G418-resistant clones with good growth were isolated and subcultured. The resultant clusters were observed and images were captured under an inverted fluorescence microscope. Subsequently, the expression of marker proteins was determined in the stably transfected cells at the third passage via immunofluorescence staining and western blot analysis.

CK3/12 and E-cadherin immunofluorescence staining. Cells cultured on sterile glass cover slips were washed briefly with PBS and fixed in a mixture of methanol and acetone for $15 \mathrm{~min}$ at room temperature. The samples were then blocked with $10 \%$ normal blocking serum in PBS for $20 \mathrm{~min}$ and incubated overnight with anti-CK3/12 or E-cadherin primary antibody. After washing 3 times with PBS, the samples were incubated in a dark chamber with fluorochrome-conjugated secondary antibody for $2 \mathrm{~h}$. The samples were extensively washed with PBS and cover slips were mounted with $90 \%$ glycerol in PBS.

Western blot analysis. Cells in each group were seeded at $4 \times 10^{5}$ cells/well into 96 -well plates. After $24 \mathrm{~h}$ of incubation, the cells were harvested, washed and lysed in $1.0 \mathrm{ml}$ of pre-cooled radioimmunoprecipitation assay buffer with freshly added protease inhibitors (Sigma-Aldrich; Merck KGaA) on ice for $30 \mathrm{~min}$ with vortexing every $10 \mathrm{~min}$. Following centrifugation at $12,000 \mathrm{x}$ g for $30 \mathrm{~min}$ at $4^{\circ} \mathrm{C}$, the supernatant was subjected to BCA protein quantification (Thermo Fisher Scientific, Inc.). Equal amounts of total protein (30 $\mu \mathrm{g}$ per lane) were subjected to $10 \%$ SDS-PAGE and transferred onto a nitrocellulose membrane (Bio-Rad Laboratories, Inc., Hercules, CA, USA), which was then blocked with $5 \%$ non-fat dry milk in Tris-buffered saline containing Tween 20 (TBS-T) for $2 \mathrm{~h}$ at room temperature. The membrane was then incubated overnight at $4^{\circ} \mathrm{C}$ with anti-PAX6 (1:500), anti-CK3/12 (1:50) and anti-E-cad (1:500). Following washing three times with
TBS-T for 20 min each, the membrane was incubated with a horseradish peroxidase-conjugated anti-rabbit or anti-mouse antibody (1:2,000; Santa Cruz Biotechnology, Inc) in 1\% bovine serum albumin (BSA; Gibco; Thermo Fisher Scientific, Inc.). Following washing as above, the membrane was incubated with enhanced chemiluminescence solution (Thermo Fisher Scientific, Inc) for $4 \mathrm{~min}$ and covered with a transparent sheet. Films were exposed and evaluated using a LAS-3000 (Fujifilm, Tokyo, Japan) imaging system.

MTT cell viability assay. The MTT assay was performed following the manufacturer's instructions. In brief, stably transfected cells were seeded in 96-well culture plates at $10^{4}$ cells/well and grown in DMEM/F12 at $37^{\circ} \mathrm{C}$ for $48 \mathrm{~h}$. Cells were incubated with MTT, and formazan crystals were solubilized in dimethyl sulfoxide (DMSO). Absorbance was measured at $570 \mathrm{~nm}$ using a Synergy 2 multidetection microplate reader (BioTek, Winooski, VT, USA). The growth curve was created based on the results.

Colony formation assay. The cells at passage 3 from each group were seeded onto 6-well plates at a density of 2,000 cells/well in three replicates. Fresh basic growth medium was added to each well following incubation for 12 days at $5 \% \mathrm{CO}_{2}$ and $37^{\circ} \mathrm{C}$. Finally, the culture medium was aspirated, each well was washed with PBS and topped up with $1 \mathrm{ml} 0.5 \%$ crystal violet solution $(0.5 \%)$, followed by incubation for $10 \mathrm{~min}$ at room temperature. Thereafter, each well was washed with a gentle stream of water under running tap water. The number of 'darkly' stained colonies was counted under a phase-contrast microscope at low magnification. Values are expressed as colony-forming efficiency calculated as the percentage of the ratio of the number of colonies counted vs. the number of cells initially seeded.

Establishment of rabbit LSCD model. A total of 10 male New Zealand white rabbits aged 6 months with an initial body weight of $2.0 \pm 0.4 \mathrm{~kg}$ were purchased from the Experimental Animal Center of Sichuan University (Chengdu, Sichuan province, China). The animals were housed at an ambient temperature of $25 \pm 2^{\circ} \mathrm{C}$ and a relative humidity of $65-70 \%$ under a $12-\mathrm{h}$ 

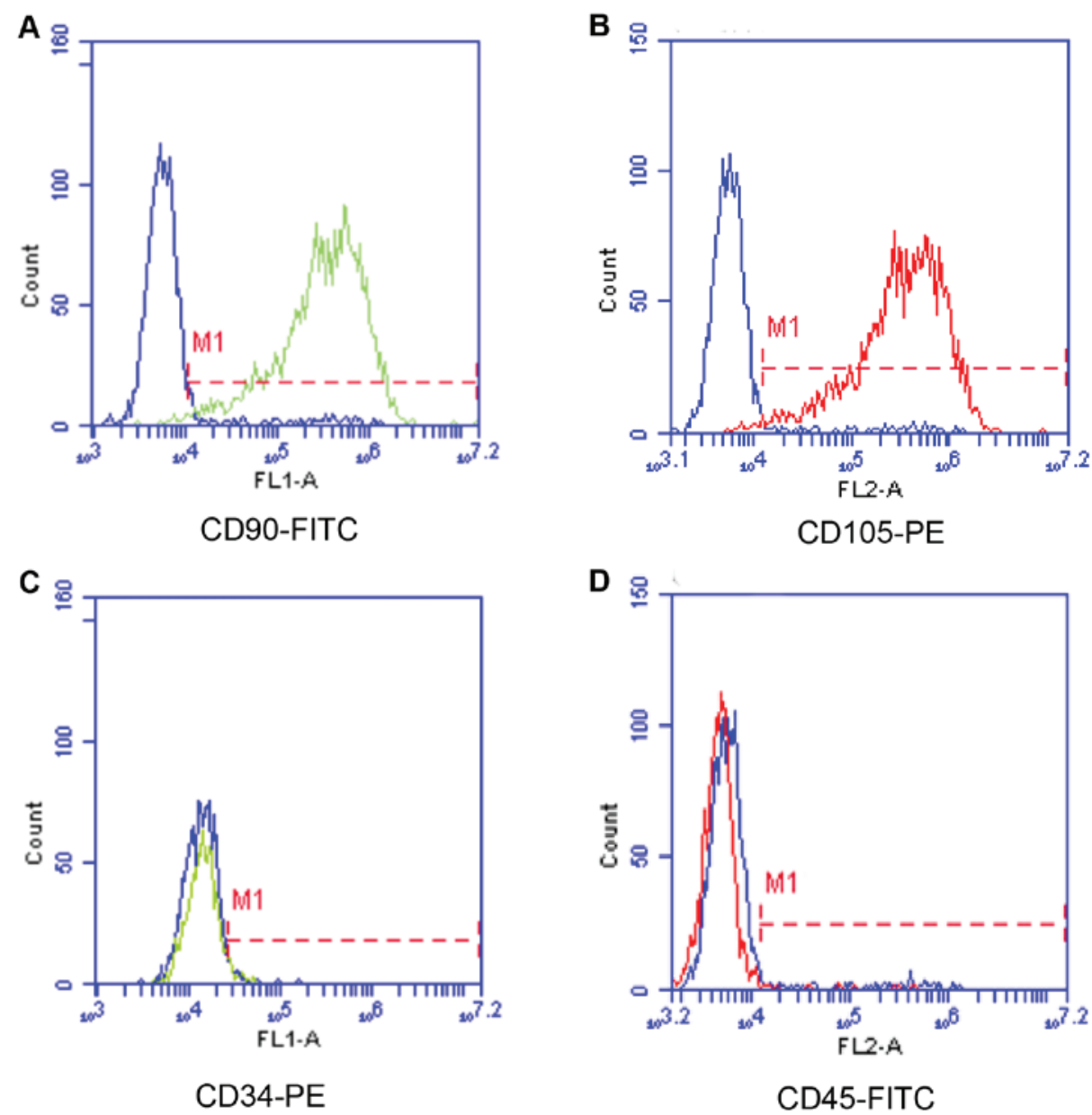

Figure 2. Flow cytometric analysis of ADMSCs. Expression of CD105, CD90, CD34 and CD45 was detected in ADMSCs isolated from bilateral inguinal fat pads of healthy Sprague Dawley rats. The cells at the third passage were incubated with FITC-labeled monoclonal antibodies against CD90 and CD45, or PE-labeled CD34 and CD105 for $30 \mathrm{~min}$ at $4^{\circ} \mathrm{C}$ and analyzed by flow cytometry to determine the surface marker expression of the ADMSCs. The control samples were not labeled with antibodies. The ADMSCs were positive for the mesenchymal stem cell markers CD90 and CD105 (A, green line; B, red line), and negative for the hematopoietic marker CD34 and CD45 (C, green line; D, red line). The blue line represents the control. ADMSCs, adipose-derived mesenchymal stem cells; FITC, fluorescein isothiocyanate; PE, phycoerythrin.

light/dark cycle. All animals were fed according to the Specific Pathogen Free Animal Criteria, and were allowed free access to food and water. All rabbits were anaesthetized with $50 \mathrm{mg} / \mathrm{kg}$ of $1 \%$ pentobarbital sodium, administered intraperitoneally. The ocular surface was briefly exposed to 5\% iodine-povidone/95\% PBS, and the left eyes were subjected to a radical $360^{\circ}$ limbal peritomy as previously described by Selver et al (21). The whole limbus with an extended dissection toward to the conjunctiva was removed with a corneal microblade, the peripheral cornea was subjected to superficial keratectomy and the central corneal epithelium was thoroughly scraped off from the cornea with scalpel blades. The post-surgical eye was treated with $0.3 \%$ levofloxacin drops, tobramycin and dexamethasone ointment 3 times a day for two weeks.

Cell transplantation. At $24 \mathrm{~h}$ after the establishment of the LSCD model, the 10 rabbits were randomly divided into the control group $(n=5)$ and transplantation group $(n=5)$, which were subjected to vehicle treatment or engraftment of EGFP-labeled PAX6 ${ }^{+}$ADMSCs, respectively. Prior to transplantation, all rabbits were anaesthetized as described above for establishing the LSCD model. The transplantation was performed based on the method by Ueno et al (20) with certain modifications. Prior to transplantation, the ADMSCs were washed twice and resuspended in $1 \mathrm{ml}$ PBS at a concentration of $1 \times 10^{7}$ cells $/ \mathrm{ml}$. Subsequently, $1 \mathrm{ml}$ PBS containing EGFP-labeled PAX6 ${ }^{+}$-transfected ADMSCs was placed into an application tube and allowed to adhere to the damaged cornea for $1 \mathrm{~h}$ in the transplantation group. In the control group, rabbits received $1 \mathrm{ml}$ PBS only. The post-surgical eye was treated with $0.3 \%$ levofloxacin drops, tobramycin and dexamethasone ointment 3 times a day for two weeks.

Histology and immunohistochemical staining. Seven days following transplantation, the 10 rabbits were anaesthetized by intraperitoneal injection of $1 \%$ pentobarbital sodium at the dose of $50 \mathrm{mg} / \mathrm{kg}$ and then sacrificed by an air embolism. The specimens used for pathological examinations were fixed in $10 \%$ formaldehyde for $24 \mathrm{~h}$ at room temperature, dehydrated using a gradual alcohol series, embedded in paraffin, and sectioned into $30 \mu \mathrm{m}$ thick sections. Subsequently, the sections were subjected to histological examination with hematoxylin and eosin (H\&E), as well as CK3/12 immunochemistry to determine the effect of cell engraftment. Corneas were fixed in formalin, embedded 

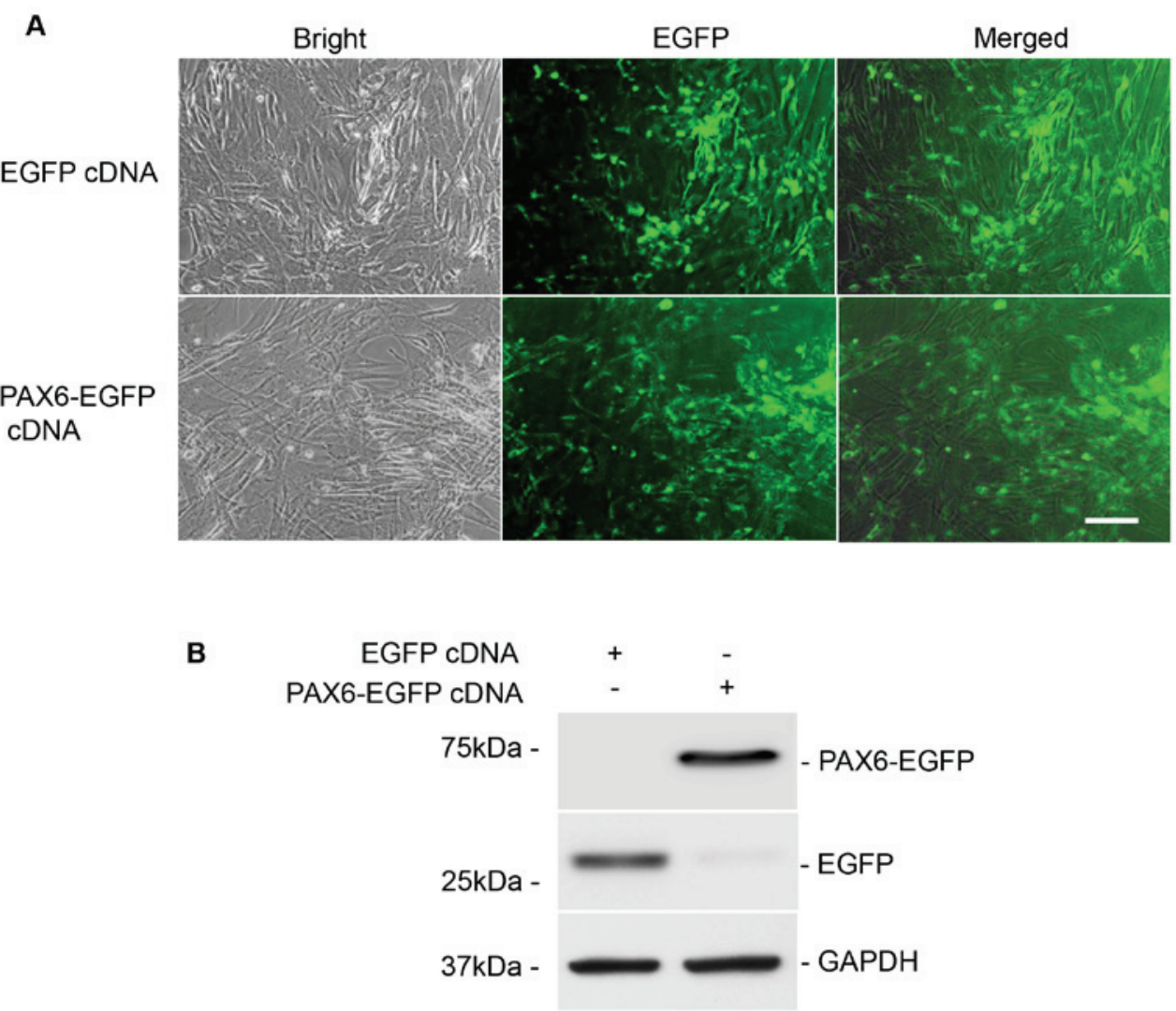

Figure 3. Stable PAX6 transfection into rat ADMSCs. (A) Fluorescence microscopic images of cultured ADMSCs with PAX6 transfection observed at 24 h after transfection. Scale bar, $100 \mu \mathrm{m}$. Left panels, phase contrast photomicrographs displaying fibroblasts assembling as uniformly long and fusiform shapes. Center panels, inverted florescence microscopy images; PAX6 expression indicated by green fluorescence was observed to be mainly located in the nuclei. Right panels, merged fluorescence and phase contrast images. (B) Validation of the transfection efficiency of EGFP and PAX6 by western blot analysis. Western blot results demonstrated that EGFP and PAX6 were present in the PAX6 expression vector-transfected ADMSCs, whereas PAX6 were absent in the control plasmid group. EGFP, enhanced green fluorescence protein; PAX6, paired box 6; ADMSCs, adipose-derived mesenchymal stem cells; cDNA, complementary DNA.

in paraffin and sectioned at $4 \mu \mathrm{m}$. For histological staining, slides were refixed, washed and stained with hematoxylin for $5 \mathrm{~min}$ and eosin for $3 \mathrm{~min}$ at room temperature, respectively. Immunostaining of the corneal sections involved deparaffinization and rehydration in PBS, permeabilization with $1 \%$ Triton $\mathrm{X}-100$ for $10 \mathrm{~min}$, blocking with $1 \% \mathrm{BSA}$ for $1 \mathrm{~h}$, overnight incubation at $4{ }^{\circ} \mathrm{C}$ with mouse anti-CK3/12 monoclonal antibody (1:100), three 5-min washes with PBS, incubation for $1 \mathrm{~h}$ with Alexa fluor-594-conjugated anti-mouse immunoglobulin $\mathrm{G}$ (1:400; cat no, A-11032, Invitrogen; Thermo Fisher Scientific, Inc.). and three 5-min washes. Sections were counterstained by incubation with $0.5 \mu \mathrm{g} / \mathrm{ml}$ DAPI for $10 \mathrm{~min}$, followed by immunofluorescence microscopy.

Statistical analysis. Values are expressed as the mean \pm standard deviation. Statistical analysis was performed using SPSS v.15.0 software for Windows (SPSS, Inc., Chicago, IL, USA). Statistical significance was assessed by a Student's t test. $\mathrm{P}<0.05$ was considered to indicate a statistically significant difference.

\section{Results}

Characterization of ADMSCs. Positive expression of the MSC markers CD105 and CD90 and negative expression of the hematopoietic lineage markers CD34 and CD45 was confirmed in third-passage ADMSCs. This result indicated the purity and successful expansion of MSCs in vitro (Fig. 2).

Stable transfection of PAX6. First, the morphology of cultured ADMSCs and the transfection efficiency with EGFP-expressing plasmids was observed by light and florescence microscopy, respectively, and the expression of Pax6 and EGFP was evaluated using western blot analysis at $24 \mathrm{~h}$ after PAX6 transfection. As expected, successful transfection of PAX6 and EGFP was observed in ADMSCs, as evidenced by green fluorescence emitted from the ADMSCs transfected with EGFP control or PAX6 expression plasmid under a florescence inverted microscope (Fig. 3A). At the same time, high expression of Pax6 was identified in the PAX6 ${ }^{+}$ADMSCs using western blot analysis (Fig. 3B). These results suggested successful and stable transfection of ADMSCs with PAX6 expression plasmid.

Differentiation of ADMSCs into corneal-like epithelial cells induced by PAX6 transfection. At $24 \mathrm{~h}$ after PAX6 transfection, ADMSCs presented as uniformly long, fusiform shaped, assembling fibroblasts (Fig. 3A). However, at 21 days following transfection, the PAX6 $6^{+}$ADMSCs exhibited a characteristic flagstone-like appearance of assembling CECs (Fig. 4A). At the same time, the expression of the corneal-specific markers 


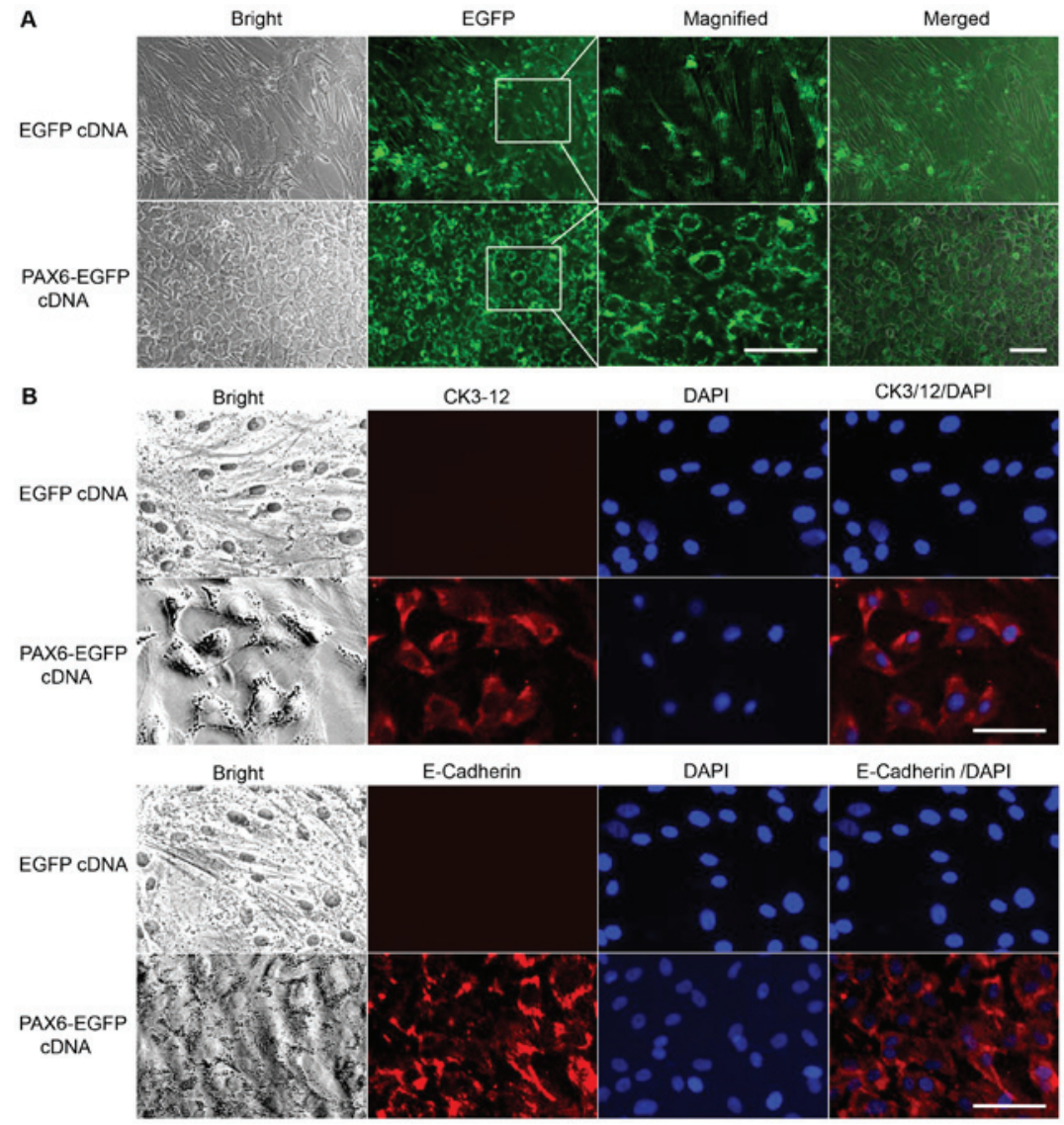

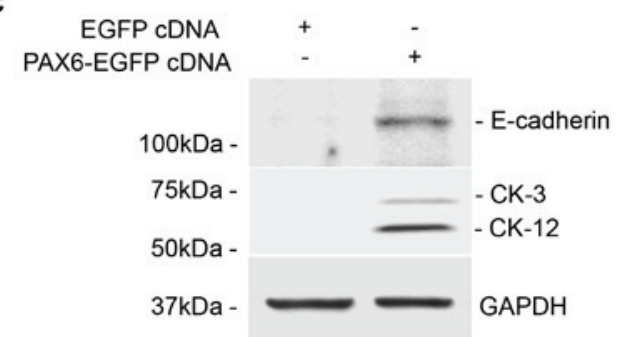

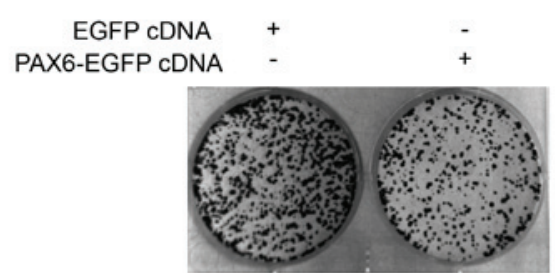

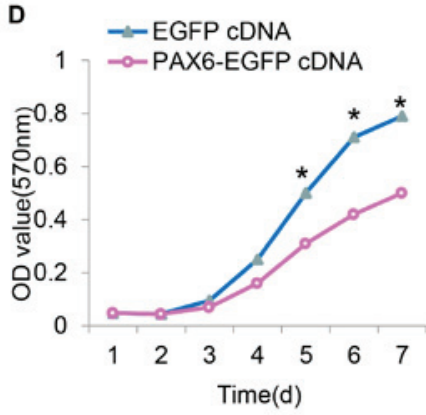

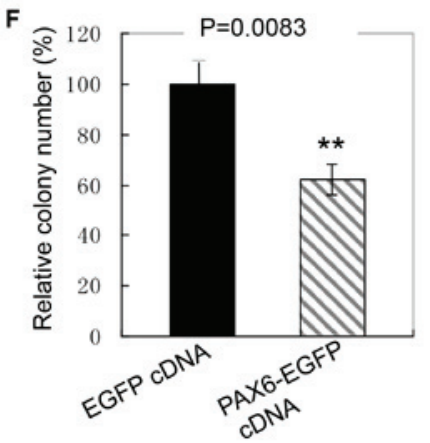

Figure 4. Characterization of the PAX6-transfected ADMSCs. (A) Fluorescence microscopic images of cultured EGFP-labeled PAX6 ${ }^{+}$ADMSCs observed at 21 days after transfection. The EGFP-labeled PAX6 $6^{+}$ADMSCs exhibited a characteristic flagstone-like appearance of assembling corneal epithelial cells. However, no obvious morphological alteration was seen in the blank plasmid group. (B) Immunofluorescence staining of corneal-specific markers CK3/12 and E-cadherin. Expression of CK3/12 and E-cadherin was observed in the PAX6-overexpressing ADMSCs (scale bars, $100 \mu \mathrm{m}$ ). (C) Western blot analysis of the corneal-specific markers CK3/12 and E-cadherin. High expression of CK3/12 and E-cadherin was revealed in the PAX6-overexpressing ADMSCs, while it was absent in ADMSCs of the blank plasmid group. (D) A cell growth curve was obtained by performing an MTT cell viability assay. (E and F) Colony formation assay of ADMSCs. The colony-forming efficiency in the PAX6 $6^{+}$ADMSCs was $\sim 60 \%$ of that in the blank plasmid group. The difference in colony-forming efficiency between the two groups was statistically significant. Values are expressed as the mean \pm standard error of the mean $(n=3)$. "P<0.05, ${ }^{* *} P<0.01$. EGFP, enhanced green fluorescence protein; PAX6, paired box 6; ADMSCs, adipose-derived mesenchymal stem cells; OD, optical density; cDNA, complementary DNA; CK, cytokeratin. 


\section{Cell transplantation operation}
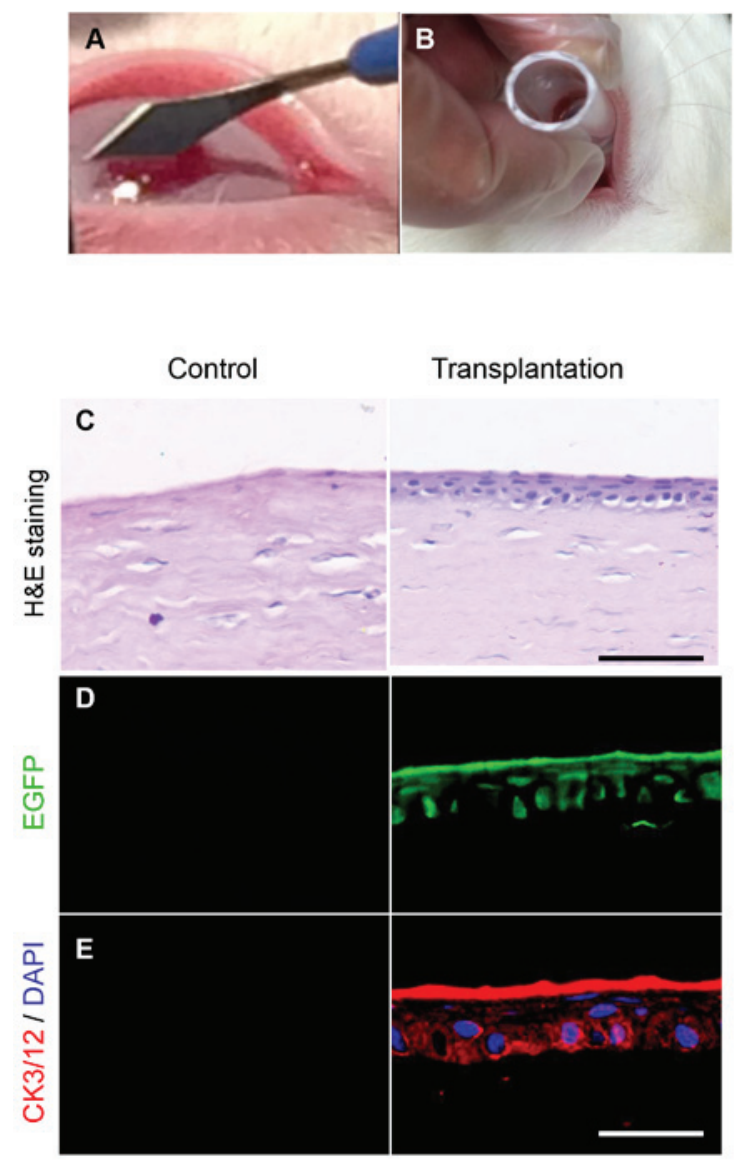

Figure 5. Cell engraftment and corneal epithelium repair in a rabbit model of limbal stem cell deficiency. (A) The left eyes were subjected to a radical $360^{\circ}$ limbal peritomy. The whole limbus with an extended dissection toward to the sclera was removed, the peripheral cornea was subjected to superficial keratectomy and the central corneal epithelium was thoroughly scraped off from the cornea. (B) The precultured EGFP-labeled PAX6 $6^{+}$-ADMSCs at a concentration of $1 \times 10^{7}$ cells $/ \mathrm{ml}$ were transplanted onto the damaged corneal surface using a small tube and allowed to adhere to the damaged cornea for $1 \mathrm{~h}$ in the transplantation group. (C-E) Seven days after transplantation, the eyeballs were excised and sectioned at $30 \mu \mathrm{m}$. The sections were subjected to histological examination with $\mathrm{H} \& \mathrm{E}$ and $\mathrm{CK} 3 / 12$ immunofluorescence staining. (C) H\&E staining demonstrated that the injured cornea transplanted with the PAX6-transfected cells featured a continuous corneal surface with stratified epithelial cells. (D and E) Immunofluorescence staining of CK3/12 in the cornea at 7 days after engraftment. The confocal images of the damaged cornea subjected to transplantation with EGFP-labeled PAX6 ${ }^{+}$-expressing ADMSCs revealed (D) attachment of EGFP-positive cells to the impaired corneal surface, with (E) concomitant expression of cornea epithelial cell-specific marker CK3/12 (scale bars, $100 \mu \mathrm{m}$ ). EGFP, enhanced green fluorescence protein; PAX6, paired box 6; ADMSCs, adipose-derived mesenchymal stem cells; H\&E, hematoxylin and eosin; CK, cytokeratin.

CK3/12 and E-cad was determined using immunofluorescence staining, which demonstrated concomitant CK3/12 and E-cad expression in the ADMSCs with PAX6 transfection (Fig. 4B). Furthermore, the expression levels of CK3/12 and E-cad were quantified by western blot analysis, which demonstrated expression of Pax6 and CK3/12 protein in the PAX6 ${ }^{+}$ ADMSCs and absence in the ADMSCs of the blank plasmid group (EGFP-labeled ADMSCs) (Fig. 4C).

The growth curve created based on the MTT cell viability assay unveiled that PAX6 overexpression significantly retarded the proliferation of PAX6-reprogrammed ADMSCs at 21 days after transfection (Fig. 4D). The colony-forming efficiency of the PAX6-reprogramed ADMSCs was 60\% that of the ADMSCs transfected with blank plasmid (Fig. 4E and F). The difference between the two groups was statistically significant $(\mathrm{P}<0.01)$.

Cell engraftment and corneal epithelium repair in a rabbit model of LSCD. In the LSCD model, the central corneal epithelium was thoroughly scraped off from the cornea and the whole limbus was removed. Seven days following transplantation of PAX6-expressing ADMSCs, the eyeballs were excised and sectioned at $30 \mu \mathrm{m}$. The sections were subjected to histological examination with $\mathrm{H} \& \mathrm{E}$ and CK3/12 immunofluorescence staining. HE staining revealed that the injured cornea transplanted with the PAX6-transfected cells demonstrated an intact morphology of the corneal surface with stratified epithelial cells (Fig. 5C). The confocal image of the damaged cornea that underwent transplantation of EGFP-labeled PAX6 $^{+}$ADMSCs revealed attachment of EGFP-positive cells to the impaired corneal surface, with concomitant expression of CEC-specific marker CK3/12. This result indicated that the grafted $\mathrm{PAX}^{+}$cells possessing the characteristics of mature CECs grew onto and reconstructed the damaged corneal surface (Fig. 5D and E).

\section{Discussion}

The integrity of the corneal surface is maintained by CECs continuously renewed by LSCs. Severe ocular disorders usually result from CEC deficiency and/or LSCD, leading to failure of epithelial regeneration or non-transparent cornea, causing corneal surface disease that leads to blindness $(22,23)$. Although allogeneic and autologous limbal transplantation are useful treatment modalities for these pathological conditions, their clinical application are currently limited due to potential complications such as corneal graft immunosuppression and impairment of the contralateral healthy eye $(5,6)$. Therefore, it is imperative to establish an alternative method for yielding sufficient seed cells for ocular surface reconstruction. MSCs have been developed as bioengineered CECs for clinical applications $(7,8)$. Thus, the present study focused on developing a technique to differentiate ADMSCs into purified CECs applicable for corneal transplantation.

PAX6 is a homeobox transcription factor acknowledged to have a critical role in corneal epithelial cell fate determination and differentiation of LSCs and CECs. Previously, Ueno et al (20) revealed that overexpression of PAX6 promoted the differentiation of mouse embryonic stem cell (ESCs) into corneal-like epithelial cells in vitro, upon transplantation onto eyes in a rabbit corneal injury model, these PAX6-reprogrammed ESCs were able to replenish CECs and repair the damaged corneal endothelial defects. Another earlier study performed by Ouyang et al (19) confirmed that PAX6 is the main driver of the differentiation of CECs and rabbit skin epithelial stem cells (SESCs) with PAX6 expression are able to transdifferentiate into corneal-like epithelial cells. These findings offer new hope for the treatment of corneal injuries, but there are still some hurdles for their clinical application. Although SESCs have priorities over ESCs due to easier 
accessibility and avoiding of ethical controversy, they continue to pose the potential risk of damaging the donor's appearance. In recent years, mesenchymal stem cells (MSCs) derived from fat and umbilical cord have been paid more attention due to the absence of the disadvantages existing in SESCs and ESCs. Thus, rat ADMSCs were used as seed cells in the present study to explore whether PAX6 gene induces the transdifferentiation of ADMSCs into corneal-like epithelial cells.

As expected, results of the present study proved for the first time that PAX6 induced ADMSCs to transdifferentiate into corneal-like epithelial cells in vitro. First, ADMSCs were transduced to stably express PAX6, as evidenced by the obvious expression of PAX6 protein in the ADMSCs after PAX6 transfection. The present study then assessed the morphological changes of ADMSCs transfected with PAX6, revealing that PAX6 ${ }^{+}$ADMSCs acquired a CEC-like morphology with a characteristic flagstone-like appearance. Furthermore, PAX6 ${ }^{+}$ ADMSCs demonstrated concomitant increases in the expression of CK3/12 and E-cadherin at 21 days after transfection. CK3 and CK12 are major intermediate filament-forming keratins of corneal epithelium, and are generally considered as the specific markers for mature CECs (24-26), and furthermore, E-cadherin is an epithelial-specific adhesion protein (27). The morphology of the PAX6 ${ }^{+}$ADMSCs mimicking that of CECs and the obvious expression of CK3/12 and E-cadherin affirmed that the ADMSCs acquired biological characteristics of CECs after PAX6 expression.

Furthermore, MTT and colony forming units assays demonstrated that the viability and proliferative capacity of the ADMSCs was retarded by PAX6 transfection, which was consistent with the results of Bardag-Gorce et al (28) and Ouyang et al (29). Collectively, these findings confirmed that the transdifferentiation of rat ADMSCs into corneal-like epithelial cells is driven by PAX6 transfection in vitro.

Following successful transfection of the PAX6 expression plasmid into rat ADMSCs using lipofection followed by G418 selection, corneal epithelial-like cells were yielded. Subsequently the EGFP-labeled PAX6 ${ }^{+}$ADMSCs were transplanted onto the damaged cornea of rabbits in an in vivo experiment. The growth and repair potential of the PAX6-reprogrammed ADMSCs for corneal epithelial defects was observed at 7 days post engraftment. Histological examination revealed that cells stably transfected with PAX6 overexpression vector were able to replenish the deficient CECs and to repair the damaged corneal surface. The abovementioned in vitro results demonstrated that the PAX6-transfected cells expressed CK3/12 and E-cadherin. In order to further evaluate whether the PAX6-transfected cells possessed the characteristics of mature CECs, CK3/12 immunofluorescence staining of corneal tissue was performed after the transplantation. Positive CK3/12 expression was observed in corneal tissue of animals that had received transplantation of EGFP-labeled PAX6 ${ }^{+}$ ADMSCs. These results suggested that the PAX6-transfected cells had similar characteristics to those of CECs in vivo. The result of the transplantation experiment also consolidated the conversion of PAX6-reprogramed ADMSCs into corneal-like epithelial cells from another perspective.

In conclusion, the results of the present study demonstrated that PAX6 induces ADMSCs to differentiate into corneal epithelial-like cells in vitro. The PAX6-reprogramed
ADMSCs were also able to reconstruct the damaged corneal surface in vivo. These findings provided evidence that ADMSCs may serve as an excellent candidate for the generation of bioengineered corneal epithelium, which holds great promise in generating a novel and encouraging paradigm for treating corneal injury. Further clarification of the ADMSC niche and the specific mechanism underlying the preferential differentiation of ADMSCs into corneal-like epithelial cells is of great significance.

\section{Acknowledgements}

This study was supported by the Scientific Research Fund of Sichuan Provincial Education Department (grant nos. 15ZA0266 and 17ZB0122), Scientific Research Project of the Health and Family Planning Commission of Sichuan Province (grant nos. 16PJ104 and 16ZD038) and Scientific Research Project of the Development and Regeneration Key Laboratory of Sichuan Province (grant nos. SYS15-004 and SYS15-008).

\section{References}

1. Yoon JJ, Ismail S and Sherwin T: Limbal stem cells: Central concepts of corneal epithelial homeostasis. World J Stem Cells 6: 391-403, 2014.

2. Cotsarelis G, Cheng SZ, Dong G, Sun TT and Lavker RM: Existence of slow-cycling limbal epithelial basal cells that can be preferentially stimulated to proliferate: Implications on epithelial stem cells. Cell 57: 201-209, 1989.

3. Frank MH and Frank NY: Restoring the cornea from limbal stem cells. Regen Med 10: 1-4, 2015.

4. Atallah MR, Palioura S, Perez VL and Amescua G: Limbal stem cell transplantation. Clin Ophthalmol 10: 593-602, 2016.

5. Rama P, Matuska S, Paganoni G, Spinelli A, De Luca M and Pellegrini G: Limbal stem-cell therapy and long-term corneal regeneration. N Engl J Med 363: 147-155, 2010.

6. Hatch KM and Dana R: The structure and function of the limbal stem cell and the disease states associated with limbal stem cell deficiency. Int Ophthalmol Clin 49: 43-52, 2009.

7. Almaliotis D, Koliakos G, Papakonstantinou E, Komnenou A, Thomas A, Petrakis S, Nakos I, Gounari E and Karampatakis V: Mesenchymal stem cells improve healing of the cornea after alkali injury. Graefes Arch Clin Exp Ophthalmol 253: 1121-1135, 2015.

8. Cejka C, Cejkova J, Trosan P, Zajicova A, Sykova E and Holan V: Transfer of mesenchymal stem cells and cyclosporine A on alkali-injured rabbit cornea using nanofiber scaffolds strongly reduces corneal neovascularization and scar formation. Histol Histopathol 31: 969-980, 2016.

9. Yun Y, Park SY, Lee HJ, Ko JH, Kim MK, Wee WR, Reger RL, Gregory CA, Choi H, Fulcher SF, et al: Comparison of the anti-inflammatory effects of induced pluripotent stem cell-derived and bone marrow-derived mesenchymal stromal cells in a murine model of corneal injury. Cytotherapy 19: 28-35, 2017.

10. Ahmad S, Stewart R, Yung S, Kolli S, Armstrong L, Stojkovic M, Figueiredo F and Lako M: Differentiation of human embryonic stem cells into corneal epithelial-like cells by in vitro replication of the corneal epithelial stem cell niche. Stem Cell 25: 1145-1155, 2007.

11. Demirayak B, Yüksel N, Çelik OS, Subaşı C, Duruksu G, Unal ZS, Yıldız DK and Karaöz E: Effect of bone marrow and adipose tissue-derived mesenchymal stem cells on the natural course of corneal scarring after penetrating injury. Exp Eye Res 151: 227-235, 2016.

12. Wilson A, Butler PE and Seifalian AM: Adipose-derived stem cells for clinical applications: A review. Cell Prolif 44: 86-98, 2011.

13. Minteer D, Marra KG and Rubin JP: Adipose-derived mesenchymal stem cells: Biology and potential applications. Adv Biochem Eng Biotechnol 129: 59-71, 2013.

14. Kasir R, Vernekar VN and Laurencin CT: Regenerative engineering of cartilage using adipose-derived stem cells. Regen Eng Transl Med1 1: 42-49, 2015. 
15. García-Villegas R, Escamilla J, Sánchez-Guzmán E Pastén A, Hernández-Quintero M, Gómez-Flores E and Castro-Muñozledo F: PAX-6 is expressed early in the differentiation of a corneal epithelial model system. J Cell Physiol 220: 348-356, 2009.

16. Collinson JM, Quinn JC, Hill RE and West JD: The roles of PAX6 in the cornea, retina, and olfactory epithelium of the developing mouse embryo. Dev Biol 255: 303-312, 2003.

17. Davis J, Duncan MK, Robison WG Jr and Piatigorsky J: Requirement for PAX6 in corneal morphogenesis: A role in adhesion. J Cell Sci 116: 2157-2167, 2003.

18. Kitazawa K, Hikichi T, Nakamura T, Sotozono C, Kinoshita S and Masui S: PAX6 regulates human corneal epithelium cell identity. Exp Eye Res 154: 30-38, 2017.

19. Ouyang H, Xue Y, Lin Y, Zhang X, Xi L, Patel S, Cai H, Luo J, Zhang M, Zhang M, et al: WNT7A and PAX6 define corneal epithelium homeostasis and pathogenesis. Nature 511: 358-361, 2014.

20. Ueno H, Kurokawa MS, Kayama M, Homma R, Kumagai Y, Masuda C, Takada E, Tsubota K, Ueno S and Suzuki N. Experimental engraftment of CECs induced by PAX6 gene transfection of mouse embryonic stem cells. Cornea 26: 1220-1227, 2007.

21. Selver OB, Durak I, Gürdal M, Baysal K, Ates H, Ozbek Z, Wang Z, Wu A and Wolosin JM: Corneal recovery in a rabbit limbal stem cell deficiency model by autologous grafts of tertiary outgrowths from cultivated limbal biopsy explants. Mol Vis 22 138-149, 2016

22. Sacchetti M, Lambiase A, Cortes M, Sgrulletta R, Bonini S Merlo D and Bonini S: Clinical and cytological findings in limbal stem cell defciency. Graefes Arch Clin Exp Ophthalmol 243 870-876, 2005
23. Lim P, Fuchsluger TA and Jurkunas UV: Limbal stem cell deficiency and corneal neovascularization. Semin Ophthalmol 24: 139-148, 2009.

24. Dua HS, Joseph A, Shanmuganathan VA and Jones RE: Stem cell differentiation and the effects of deficiency. Eye (Lond) 17 877-885, 2003.

25. Elder MJ, Hiscott P and Dart JK: Intermediate filament expression by normal and diseased human corneal epithelium. Hum Pathol 28: 1348-1354, 1997.

26. Jeon S, Choi SH, Wolosin JM, Chung SH and Joo CK: Regeneration of the corneal epithelium with conjunctival epithelial equivalents generated in serum- and feeder-cell-free media. Mol Vis 19: 2542-2550, 2013

27. Lyngholm M, Vorum H, Nielsen K, Østergaard M, Honoré B and Ehlers N: Differences in the protein expression in limbal versus central human corneal epithelium-a search for stem cell markers. Exp Eye Res 87: 96-105, 2008.

28. Bardag-Gorce F, Hoft RH, Wood A, Oliva J, Niihara H, Makalinao A, Thropay J, Pan D, Meepe I, Tiger K, et al: The role of E-cadherin in maintaining the barrier function of corneal epithelium after treatment with cultured autologous oral mucosa epithelial cell sheet grafts for limbal stem deficiency. J Ophthalmol 2016: 4805986, 2016.

29. Ouyang J, Shen YC, Yeh LK, Li W, Coyle BM, Liu CY and Fini ME: PAX6 overexpression suppresses cell proliferation and retards the cell cycle in corneal epithelial cells. Invest Ophthalmol Vis Sci 47: 2397-2407, 2006.

(i) (9) This work is licensed under a Creative Commons Attribution-NonCommercial-NoDerivatives 4.0 International (CC BY-NC-ND 4.0) License. 